\title{
Vertebroplasty: Review of the Procedure in Relation to Patients with Fracture of Osteoporotic Origin
}

\author{
Iñaki Arrotegui* \\ Department of Neurosurgery, Hospital General Universitario de Valencia, Spain
}

*Corresponding author: Iñaki Arrotegui, Department of Neurosurgery, Consultant Neurosurgeon Hospital General, Universitario de Valencia, Avda Tres Cruces S/n 46014- Valencia, Spain

Submission: May 29, 2018; Published: August 13, 2018

\section{Introduction}

Vertebroplasty was initially described by Gallibert and cols. in 1987 to treat symptomatic hemangiomas of the vertebral body [1].

Vertebroplasty and kyphoplasty are procedures that are used to treat painful vertebral compression fractures in the spine, which are a common consequence of osteoporosis. After vertebroplasty, approximately $75 \%$ of patients recover lost mobility and become more active Vertebroplasty and kyphoplasty are minimally invasive procedures for the treatment of painful vertebral compression fractures (FCV), fractures that involve the vertebral bodies that make up the spine [2].

When a vertebral body fractures, the normal rectangular shape of the bone is transformed into a compressed form, causing pain. These compression fractures can include the collapse of one or more vertebrae in the spine and are a common consequence of osteoporosis. Osteoporosis is a disease that results in the loss of normal bone density, mass, and strength, leading to a condition in which the bones become more porous and vulnerable to fracture easily. The vertebra can also be weakened by cancer [3].

For vertebroplasty, imaging guidance, usually fluoroscopy, is used to inject a mixture of cement into the fractured bone through a hollow needle. During a kyphoplasty, a balloon is first inserted into the fractured bone, through the hollow needle, to create a cavity or space. The cement is injected into the cavity after the balloon is removed.

Vertebroplasty and kyphoplasty are used to treat painful spinal compression fractures in the spine, often caused by osteoporosis. Usually, vertebroplasty and kyphoplasty are recommended after attempts to less invasive treatments, such as bed rest, a back support or analgesics have been ineffective. Vertebroplasty and kyphoplasty can be performed immediately in patients with problematic pain requiring hospitalization, or with conditions that limit bed rest and the use of medications.

Vertebroplasty and kyphoplasty are also performed in patients who: they are old or weak and whose bones will not recover completely from a fracture have vertebral compression due to a malignant tumor suffer from osteoporosis caused by treatment with long-term steroids or a metabolic disorder. Kyphoplasty and vertebroplasty should be completed within eight weeks after the acute fracture occurs so that the treatment has the highest probability of being successful [4].

When a vertebra breaks or fractures, bone fragments are created. Pain occurs when these fragments slip or rub against each other, or protrude into the spinal cord. Vertebroplasty involves injecting the bone with a cement mix to fuse the fragments, strengthen the vertebra and relieve pain. First, the skin is numbed with a local anesthetic. Then, using the imaging guide, the hollow needle or trocar is passed through the skin and into the vertebral body to inject the cement mixture into the vertebra. In kyphoplasty, after numbing the skin locally, a balloon is inserted through the trocar, into the fractured vertebra, where it is inflated to create a cavity. The balloon is removed before the cement is injected into the cavity created by the balloon.

The minimally invasive procedures, guided by images, such as vertebroplasty and kyphoplasty, are performed mostly by an interventional radiologist, orthopedic surgeon, neurosurgeon, specially trained. This procedure is often done in outpatients. However, some patients may need hospitalization after the procedure. Please consult your doctor about whether you will be admitted or not. During the procedure, it could be connected to monitors that control the heartbeat, blood pressure, and pulse. With X-ray guidance, the trocar is passed through the vertebral muscles, until its tip is positioned precisely within the fractured vertebra. In vertebroplasty, the orthopedic cement is then injected. The medical-grade cement hardens quickly, typically within 20 minutes. The trocar is removed after injecting the cement.

Pressure will be applied to prevent any bleeding, and the opening made in the skin is covered with a dressing. No suturing is necessary. This procedure is typically completed within 45 minutes. It may last longer if you are treating more than one vertebra. Vertebroplasty and kyphoplasty can increase a patient's functional abilities and allow them to return to a previous level of 
activity without any form of physical therapy or rehabilitation [5]. These procedures are usually successful in relieving pain caused by a vertebral compression fracture; Many patients feel relief almost immediately or within a few days. Most patients are free of symptoms. After vertebroplasty, approximately $75 \%$ of patients recover lost mobility and become more active, which helps to combat osteoporosis. After the procedure, patients who had been immobilized can get out of bed, and this can help reduce the risk of pneumonia. Greater activity strengthens the muscles, and this further encourages mobility.

In general, vertebroplasty and kyphoplasty are safe and effective procedures. No surgical incision is needed: just a small cut on the skin that does not need stitches [6].

According to the European Study on Osteoporosis Vertebral

The fractures are distributed:
A. Wedging $(51 \%)$
B. Biconcave $(17 \%)$
C. Crushing $(13 \%)$
D. Wedging and crushing (7\%)
E. Biconcave wedges and vertebrae $(6 \%)$
F. Crushing and biconcavities (2\%)
G. Combination of the three (4\%)

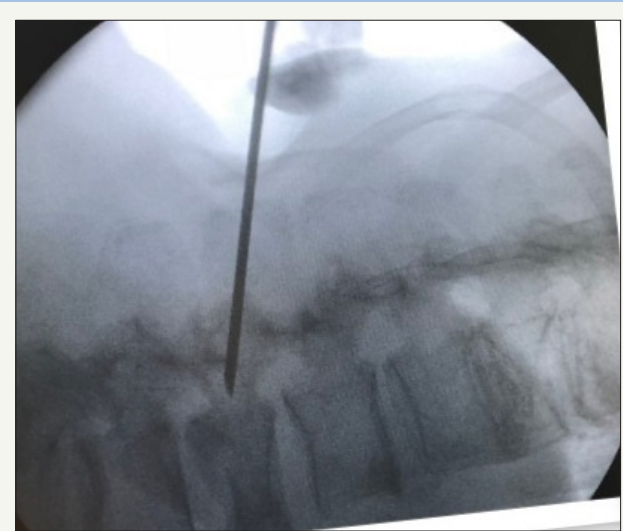

Figure 1.

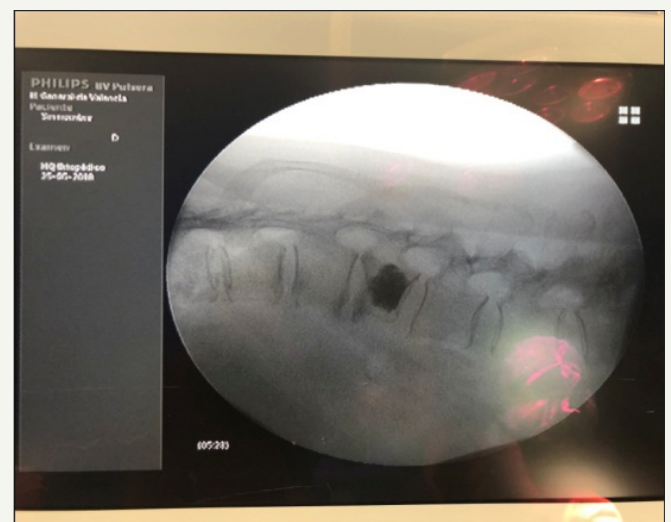

Figure 2.
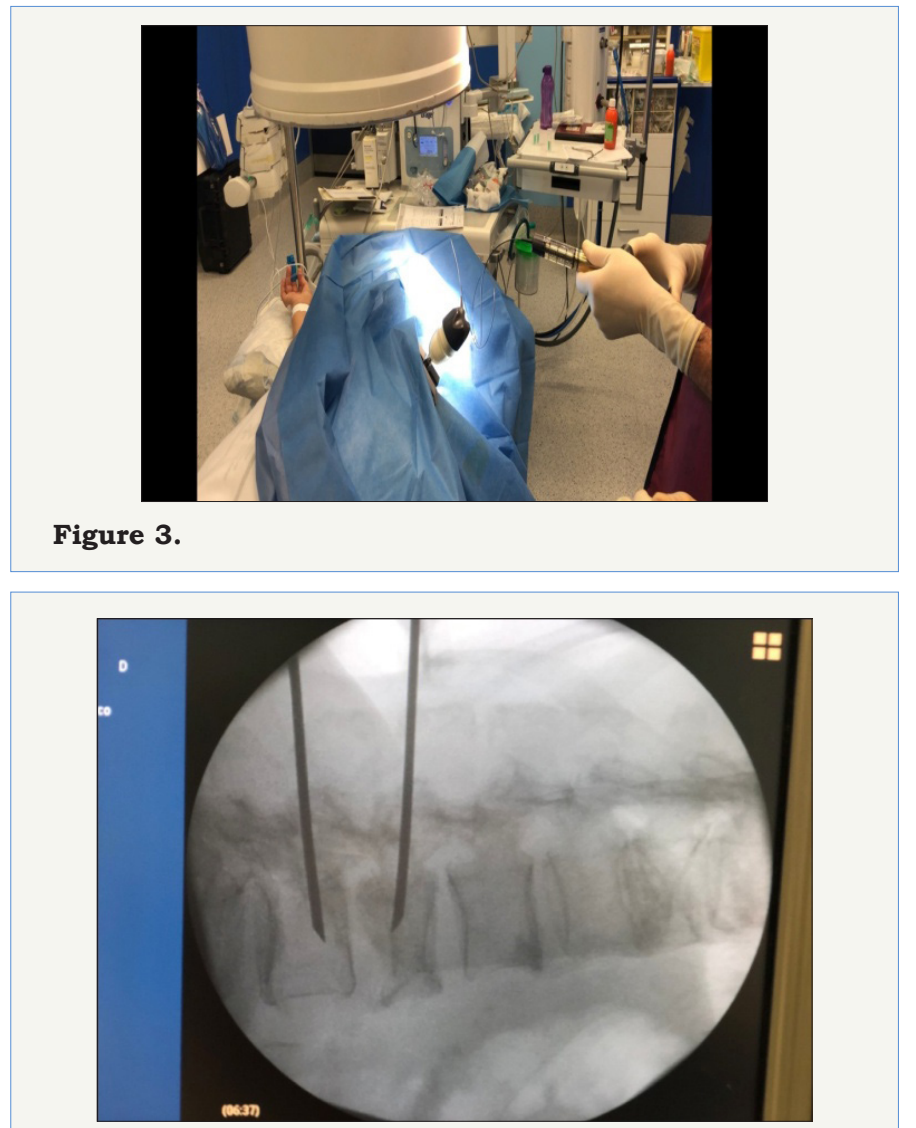

Figure 4.

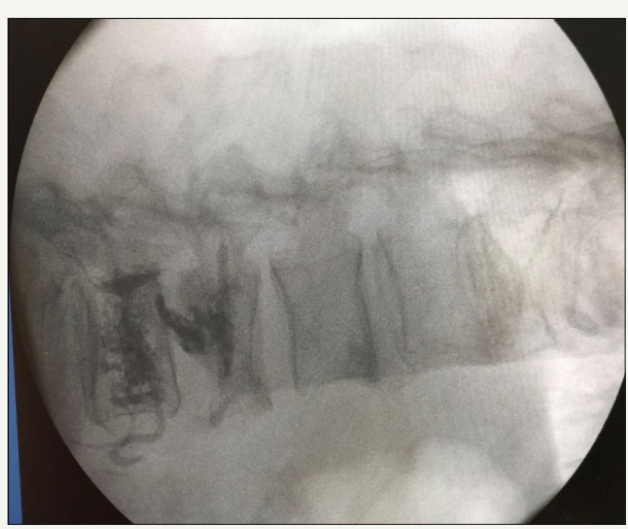

Figure 5.

The two patients with symptomatic vertebral fracture have severe back pain after a traumatism or a bad gesture. The pain worsened with the erect position and presented a kyphotic postural attitude in standing. The attached Rx Figure 1-4, shows the location of the osteoporotic fractures in T11 and L3 and L4, the cementation system 3 and the cementation of both cases Figure 2-5. After resting for 30 minutes with neurological assessment and monitoring, and when there were no problems, the patients were discharged [7].

\section{References}

1. Esses SI (2010) The treatment of symptomatic osteoporotic spinal compression fractures: guideline and evidence report. American Academy of Orthopaedic Surgeons. pp. 1-194. 
2. Doidge J, Merlin T, Liufu Z, Tamblyn D, Jia LY (2011) Review of interim funded service: Vertebroplasty and new review of kyphoplasty . Medical Services Advisory Committee.

3. (2013) Percutaneous vertebroplasty and percutaneous balloon kyphoplasty for treating osteoporotic vertebral compression fractures. NICE The National Institute for Health and Care Excelence, p. 3.

4. Nicole B, Paul Lynch MD, Tory Mc Junkin MD (2008) Vertebroplasty and Kyphoplasty.

5. Bliemel C, Oberkircher L, Buecking B, Timmesfeld N, Ruchholtz S, et al. (2012). Higher incidence of new vertebral fractures following percutaneous vertebroplasty and kyphoplasty-fact or fiction? Actaorthopaedica Belgica 78(2): 220-229.

6. Wang LJ, Yang HL, Hi YX, Jiang WM, Chen L (2012) Pulmonary cement embolism associated with percutaneous vertebroplasty or kyphoplasty: a systematic review. Orthopaedic surgery 4(3): 182-189.

7. Wardlaw D, Van Meirhaeghe (2012) Balloon kyphoplasty in patients with osteoporotic vertebral compression fractures, Expert Review of Medical Devices 9(4): 423-436.
For possible submissions Click Here Submit Article

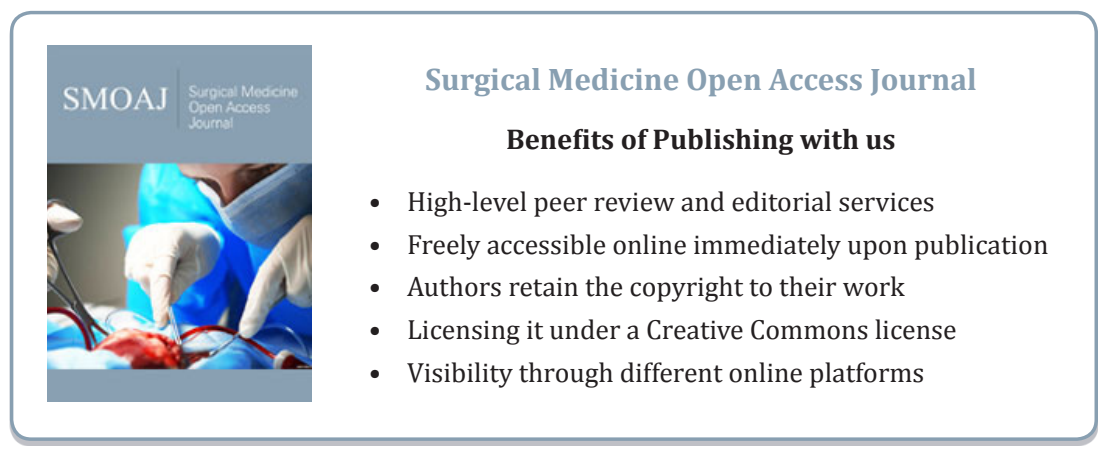

